\title{
Der soziale Ausgleich in der gesetzlichen Krankenversicherung
}

\author{
Peter F. Lutz/Ulrike Schneider
}

Diskussionspapier Nr. 200

März 1997

ISSN 0949 - 9962

Fassung vom 15. Juni 1998

\author{
Dr. Peter F. Lutz \\ Dr. Ulrike Schneider \\ Universität Hannover \\ Institut für Volkswirtschaftslehre \\ Königsworther Platz 1 \\ D-30167 Hannover \\ lutz@mbox.vwl.uni-hannover.de \\ schneider@mbox.vwl.uni-hannover.de
}




\section{Inhaltsverzeichnis}

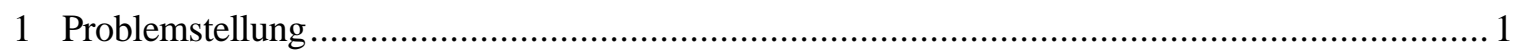

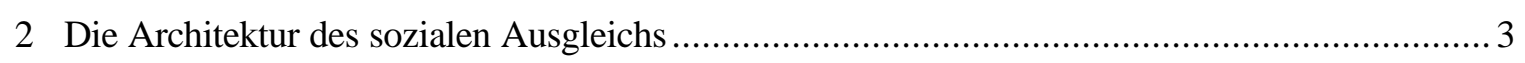

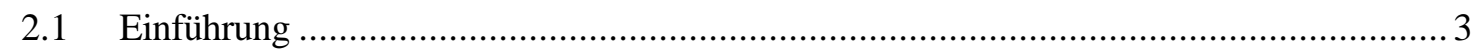

2.2 Der Versicherungszwang als Konstruktionsgrundlage .......................................... 4

2.3 Über- und Unterschreitungen des äquivalenten Beitrags ........................................... 5

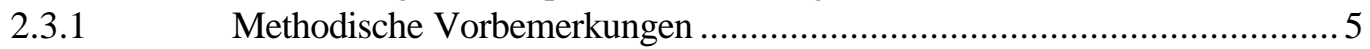

2.3.2 Äquivalenz bei beitragsabhängigen Leistungen ................................... 8

2.3.3 Äquivalenz bei beitragsunabhängigen Leistungen ............................... 10

2.3.4 Äquivalenz für den Gesamtversicherungsschutz.................................. 11

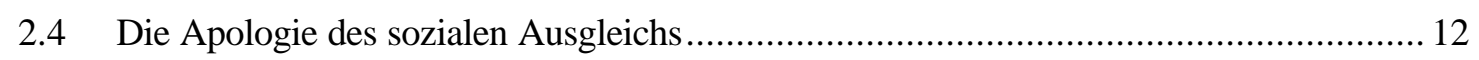

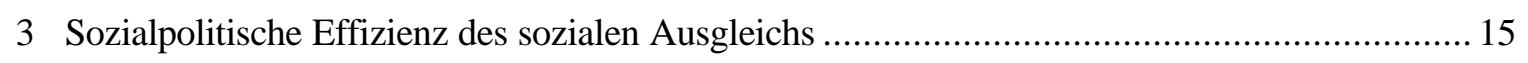

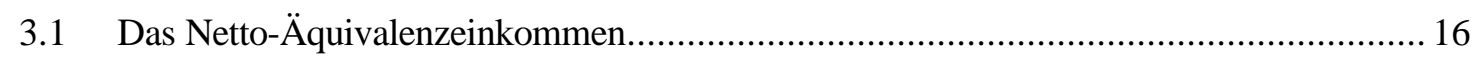

3.2 Die Nettoposition als Indikator für die Stellung im sozialen Ausgleich ......................... 18

3.3 Empirischer Zusammenhang zwischen Netto-Äquivalenzeinkommen und

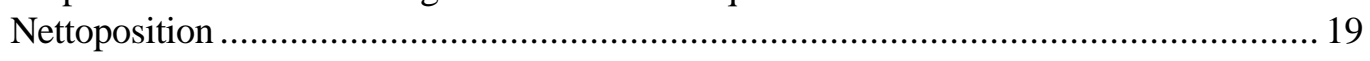

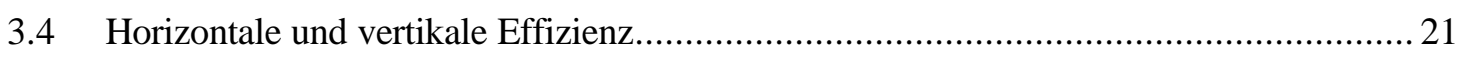

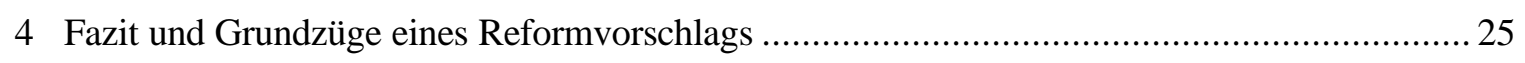




\section{Problemstellung}

Während der Umfang des Versicherungsschutzes durch die gesetzliche Krankenversicherung vom Krankengeld einmal abgesehen - für alle Versicherten identisch ist, werden dafür sehr unterschiedliche Beiträge erhoben. Für die Beitragshöhe ist nicht das Krankheitsrisiko, sondern sind die risikounabhängigen Beitragsbemessungsprinzipien der gesetzlichen Krankenversicherung maßgebend. Da die gesetzliche Krankenversicherung mangels Bundeszuschüssen mittelfristig mit einem ausgeglichenen Budget arbeiten muß, folgt hieraus, daß einige Versicherte einen geringeren, andere einen höheren als den versicherungstechnisch äquivalenten Beitrag entrichten müssen. Für diese Abweichungen wird oft der Begriff des sozialen Ausgleichs verwendet. Die Höhe der jeweiligen individuellen Abweichungen ist dabei in der Sozialstaatspraxis nicht bekannt, weil ein äquivalenter Beitrag nicht berechnet wird. Über Begünstigung oder Zahlerrolle entscheiden nach wie vor die Prinzipien aus der Konzeptionsphase der gesetzlichen Krankenversicherung, insbesondere die proportional arbeitsentgeltabhängige Beitragsbemessung sowie die vom Haushaltseinkommen unabhängige beitragsfreie Mitversicherung von Familienangehörigen. Ein Ziel dieses Beitrags ist es, ausgehend von der personalen Inzidenz dieser Regelungen, zu prüfen, wie hoch die sozialpolitische Effektivität und Effizienz eines so konzipierten sozialen Ausgleichs ist.

Alle Nichtmitglieder der gesetzlichen Krankenversicherung werden von der Finanzierung der Begünstigungen ausgenommen. Durch die Heranziehung nur einer bestimmten Bevölkerungsgruppe zu ihrer Aufbringung entsteht ein verfassungsrechtliches Problem. Aus Art. 3 Abs. 1 GG kann für das Abgabenrecht das Prinzip der Belastungsgleichheit (Lastengleichheit) abgeleitet werden, das durch die Rechtsprechung des Bundesverfassungsgerichtes konkretisiert wurde. Es erfordert für alle "Sonderlasten", d.h. Zwangsabgaben, die nicht gleichmäßig von allen Bürgern nach dem Prinzip der Leistungsfähigkeit erhoben werden, eine besondere Rechtfertigung. ${ }^{1}$ Damit werden dem Staat Schranken bei der Umgehung der Steuerfinanzierung und der willkürlichen Heranziehung nur bestimmter gesellschaftlicher Gruppen zur Staatsfinanzierung gesetzt. Die Sozialversicherungsbeiträge und damit auch die Beiträge zur gesetzlichen Krankenversicherung stellen nach Rechtsprechung des Bundesverfassungsgerichts solche Sonderlasten dar und bedürfen dementsprechend einer besonderen Rechtfertigung. ${ }^{2}$ So-

\footnotetext{
Vgl. z.B. BVerfG 10.12.1980 E55 274 (303f), 23.1.1990 E 81, 156 (185).
}

Vgl. dazu BVerfG 23.1.1990 E 81, S. 156. 
weit dem Krankenversicherungsbeitrag eine ökonomisch äquivalente Gegenleistung gegenübersteht, gilt er, dem Sozial- und Verfassungsrechtswissenschaftler F. Kirchhof folgend, als verfassungsrechtlich unproblematisch. ${ }^{3}$ Bei nicht mehr durch das Äquivalenzprinzip gedeckten Überbelastungen von Versicherten in der GKV wird jedoch ein Widerspruch zum Gleichheitssatz, dem die Rechtsprechung noch nicht hinreichend nachgegangen sei, von Kirchhof anerkannt. ${ }^{4}$ Neben der verfassungsrechtlichen Frage gibt es ein weiteres Motiv, den sozialen Ausgleich genauer zu untersuchen: Seine sozialpolitische Effizienz im heutigen Umfeld ist keineswegs sicher. Insbesondere ist zu prüfen, inwieweit er sogenannte Streuverluste verursacht. Damit besteht auch eine Beziehung zu der Höhe der Personalnebenkosten und deren Implikationen für den Arbeitsmarkt.

Quantitative Relevanz kommt dem Problem aufgrund der Tatsache zu, daß es bei den Beiträgen zur gesetzlichen Krankenversicherung um ganz erhebliche Finanzvolumina geht. Die bei den personalintensiven Gesundheitsleistungen nur unterdurchschnittlich steigerbare Arbeitsproduktivität bei gleichzeitig weitgehend durchschnittlicher oder sogar überdurchschnittlicher Lohn- und Gehaltsentwicklung ("Baumolsche Kostenkrankheit"), medizinische und medizinisch-technische Fortschritte sowie weitere Faktoren haben das Haushaltsvolumen der gesetzlichen Krankenversicherung ganz erheblich anwachsen lassen. Mit Einnahmen von 256 Mrd. DM und Ausgaben von 272 Mrd. DM im Jahr 1996 ist der Haushalt der gesetzlichen Krankenversicherung bereits mehr als halb so groß wie der Bundeshaushalt (Einnahmen: 423 Mrd. DM, Ausgaben: 496 Mrd. DM). ${ }^{5}$

Im weiteren wird nach einem Schlaglicht auf die historische Genese zunächst der wenig konkrete Begriff des sozialen Ausgleichs präzisiert (Kapitel 2). Unser Ansatz weicht hier von bisherigen Ansätzen ${ }^{6}$ zu diesem Thema deutlich ab, da er den Leistungsrisikoausgleich von den übrigen Umverteilungswirkungen der gesetzlichen Krankenversicherungen trennt. Das Leistungsrisiko einer Krankenversicherung variiert mit drei Faktoren: Alter, Geschlecht und Gesundheitszustand. Beim Ausgleich des Leistungsrisikos durch eine Durchschnittsprämie handelt es sich um eine verfassungsrechtlich unumstrittene Umverteilung, die - mit Ausnahme des Geschlechterausgleichs - auch im langfristigen privaten Krankenversicherungskontrakt anzutreffen

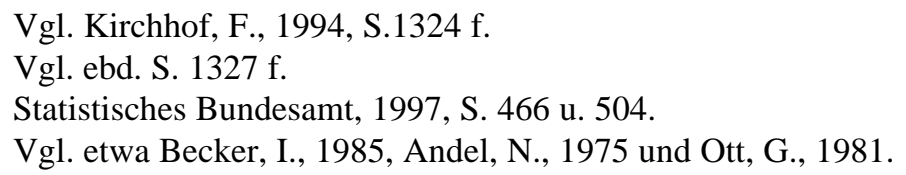


ist. So trägt das Versichertenkollekiv bei einem nach deutschem Versicherungsvertragsrecht versicherten jungen Menschen prinzipiell lebenslang sowohl das Risiko etwaiger Verschlechterungen des Gesundheitszustandes und gleicht - im Wege von retrospektiv kalkulierten Alterungsrückstellungen - das altersabhängig unterschiedliche Krankheitsrisiko intertemporal aus. Nur darüber hinausgehende Umverteilungen sind versicherungsfremd und werden von uns unter den Begriff des sozialen Ausgleichs gefaßt.

Insbesondere wird die sozialpolitische Effizienz dieses Ausgleichs überprüft. Dazu müssen sein Umfang und die personale Zahlungsinzidenz mit Hilfe von Daten aus dem Sozio-ökonomischen Panel (SOEP) ermittelt werden (GP 3). Aus dieser Analyse ergibt sich schließlich, in welchem Maße eine Finanzreform die gesetzliche Krankenversicherung gleichheitskonform sowie beschäftigungs- und sozialpolitisch effizienter gestalten könnte (GP 4).

\section{Die Architektur des sozialen Ausgleichs}

\section{$2.1 \quad$ Einführung}

Die gesetzliche Krankenversicherung finanziert sich durch Beiträge und sonstige Einnahmen. Sonstige Einnahmen sind z.B. Vermögenserträge und Einnahmen aus Ersatz- und Erstattungsansprüchen. Sonstige Einnahmen werden im folgenden als unproblematisch ausgeklammert und sind überdies quantitativ wenig relevant. ${ }^{7}$ Hauptfinanzierungsinstrument ist der Beitrag, der meist proportional arbeitsentgeltabhängig bis zu einer Beitragsbemessungsgrenze von jedem Mitglied einer gesetzlichen Krankenkasse erhoben wird. ${ }^{8}$ Mitglieder einer gesetzlichen Krankenkasse und damit beitragspflichtig waren im Jahr 1996 mit 50,8 Millionen ca. 62 v.H. der 81,9 Millionen Einwohner Deutschlands. ${ }^{9}$ Die Mehrzahl dieser Mitglieder war versicherungspflichtig, hatte also keine Möglichkeit, der Beitragspflicht durch Austritt aus der gesetzlichen Krankenversicherung zu entgehen. Es wird somit ein bestimmter Personenkreis innerhalb der Gesamtbevölkerung mit einer Zwangsabgabe belegt.

7 So belief sich 1991 der Anteil der sonstigen Einnahmen an den Gesamteinnahmen auf 3,6 v.H. In den letzten Jahren ist dieser Anteil in den Statistiken angestiegen, die auch Zahlungen aus dem Risikostrukturausgleich ausweisen. Doch handelt es sich dabei lediglich um eine Umverteilung bestehender Einnahmen.

8 Die Beitragssätze lagen im Jahr 1995 zwischen 9 und 15,5 v.H. Die Beitragsbemessungsgrenze lag 1995 monatlich bei DM 5.850 (West) und DM 4800 (Ost).Zu den Details der Beitragsberechnung vgl. $\S 220 \mathrm{ff}$. SGB V.

9 Vgl. Statist. Jahrbuch 1997, S. 466, 46. Man beachte, daß weit mehr als 62\% der Bevölkerung gesetzlich krankenversichert sind, da für Mitglieder die Möglichkeit besteht, Familienangehörige beitragsfrei mitzuversichern. 


\subsection{Der Versicherungszwang als Konstruktionsgrundlage}

Bei der Einführung der gesetzlichen Krankenversicherung im vorigen Jahrhundert ging es darum, die soziale Lage der Arbeiterschaft zu verbessern. Dies kommt in der sogenannten "Kaiserlichen Botschaft" aus dem Jahre 1881 zum Ausdruck, in der angekündigt wird, "(..) die Heilung der sozialen Schäden nicht ausschließlich im Wege der Repression sozialdemokratischer Ausschreitungen, sondern gleichmäßig auf dem der positiven Förderung des Wohls der Arbeiter zu suchen (..)". ${ }^{10}$ Ob dieses Ziel aus Gründen des Machterhalts oder anderen Gründen verfolgt wurde, kann hier dahingestellt bleiben. Durch das Krankenversicherungsgesetz des Jahres 1883 wurde für weite Teile der Arbeiterschaft ein Versicherungszwang geschaffen. Dies stellte sicher, daß kein Arbeiter sein niedriges Einkommen für andere Zwecke verausgabte, sich nicht versicherte und deshalb zusammen mit seiner Familie bei Krankheit zum sozialen Problem werden konnte. Diejenigen Personen, die anderweitig abgesichert waren, wurden von der Versicherungspflicht ausgenommen. Dies waren z.B. die Beamten, deren Krankheitskosten vom Staat übernommen wurden.

Auch heute noch ist ein gewisser Personenkreis pflichtversichert (§ 5 SGB V). Die Pflichtversicherung wird nun oft mit der "Schutzbedürftigkeit" dieser Personengruppe begründet. ${ }^{11}$ Diese Schutzbedürftigkeit könnte auf mangelnder Einsicht in die Notwendigkeit eines Krankenversicherungsschutzes oder auf mangelnde Fähigkeit zur Finanzierung eines solchen oder beidem gründen. In jedem Fall ist jedoch bei funktionierenden Märkten für Versicherungsschutz die Pflichtversicherung in der gesetzlichen Krankenversicherung nicht das schonendste Mittel zur Verwirklichung eines Schutzzwecks. Dem Subsidiaritätsprinzip würde es vielmehr entsprechen, dem Versicherten die Möglichkeit zu geben, sein Krankheitskostenrisiko privat abzusichern. Die Versicherten könnten dann zwischen verschiedenen im Preiswettbewerb stehenden Versicherungsangeboten wählen. Um der „Schutzbedürftigkeit“ Rechnung zu tragen, bräuchte der Staat nur eine generelle Versicherungspflicht zu schaffen. Eine solche Form der Versicherungspflicht sichert bekanntlich seit langem den Versicherungsschutz im Bereich der Kraftfahrzeug-Haftpflicht und wird von verschiedenen Gesundheitsökonomen auch für die Krankenversicherung vorgeschlagen. ${ }^{12}$

10 zitiert nach: Schlenker, R.-U., 1994, S.5.

11 Vgl. z.B. Gesellschaft für Versicherungswissenschaften und -gestaltung e.V., 1992, S.15.

12 Vgl. Wagner, G., 1997, S. 106 m.w.N. 
An der bisherigen Form der Pflichtversicherung muß jedoch festgehalten werden, wenn ein sozialer Ausgleich praktiziert werden soll. Es gibt in der GKV eine noch näher zu bestimmende Personengruppe, deren Mitglieder höhere Beiträge zahlen, als dies durch den Wert ${ }^{13}$ des ihnen gewährten Versicherungsschutzes gerechtfertigt wäre. Mit den sich jeweils ergebenden Mehrbeträgen wird der soziale Ausgleich in der GKV, z.B. die beitragsfreie Mitversicherung von Familienangehörigen, finanziert. Mitglieder dieser Gruppe von Überzahlern hätten einen ökonomischen Anreiz, sich anderweitig zu einem günstigeren Beitrag zu versichern. Würde die Pflichtversicherung in der GKV durch einen Versicherungsnachweis ersetzt, wäre zu befürchten, daß viele dieser Personen aus der GKV ausscheiden würden und infolgedessen der Beitragssatz in die Höhe schnellt.

\section{3 Über- und Unterschreitungen des äquivalenten Beitrags}

\subsubsection{Methodische Vorbemerkungen}

Um den sozialen Ausgleich zu quantifizieren, muß zunächst geklärt werden, welchen Wert der durch die GKV gewährte Versicherungsschutz hat. Zunächst könnte man an eine Marktbewertung denken, doch wird das Produkt „Krankenversicherungsschutz in der GKV“ nicht auf Wettbewerbsmärkten gehandelt. Die am ehesten vergleichbaren privaten Krankeitskostenvollversicherungen zeichnen sich durch einen abweichenden Leistungsumfang aus. So werden ärztliche Leistungen dort z.B. mit anderen Sätzen honoriert. Außerdem werden private Krankheitskostenvollversicherungen so kalkuliert, daß die Prämie über die gesamte Vertragsdauer konstant bleiben soll. Die Prämie ist deshalb vom Eintrittsalter abhängig. Die je nach Anbieter überdies immer noch recht unterschiedlichen Prämien für private Krankenversicherung stellen somit keinen geeigneten Indikator für den ökonomischen Wert des Krankenversicherungsschutzes in der GKV dar.

In diesem Beitrag wird deshalb ein anderer Ansatz gewählt. Der Wert des Versicherungsschutzes wird als Erwartungswert der Versicherungsleistungen einschließlich der gleichmäßig auf jeden Kontrakt verteilten Verwaltungskosten (loading costs) definiert. Dieser Wert wird dann mit der tatsächlichen Höhe des Beitrags abgeglichen. Übersteigt der tatsächliche Beitrag den Erwartungswert des Versicherungsschutzes zuzüglich der anteiligen Verwaltungskosten, so

\footnotetext{
13 Wir äußern uns später zum Bewertungsproblem.
} 
wird im folgenden von einer Überbelastung des Versicherten gesprochen, umgekehrt von einer Unterbelastung.

In der Literatur zur Krankenversicherung wird oft eine Risikodifferenzierung bei der Berechnung von äquivalenten Beiträgen vorgenommen. ${ }^{14}$ Dazu werden verschiedene Risikoklassen gebildet, meist differenziert nach Geschlecht und Alter, oft auch nach Vorerkrankungen. Für jede dieser Risikoklassen ergeben sich andere Erwartungswerte der Versicherungsleistungen, mithin jeweils andere äquivalente Prämien. Im Ergebnis müssen Vorerkrankte, Frauen und Ältere höhere Prämien bezahlen. Je mehr individuelle Äquivalenz hergestellt wird, desto geringer ist die versicherungsimmanente Umverteilung. Die Prämie nähert sich dem Barwert der zukünftigen Leistungen an und die Versicherung gerät immer mehr zum individuellen Ansparvorgang für spätere Ausgaben. Die Risikodifferenzierung führt letztlich weg vom Versicherungsprinzip hin zu einer Risikovorsorge durch Vermögensbildung. Wenn, um ins theoretische Extrem zu gehen, dem Versicherer der Zeitpunkt des Auftretens und die Kosten aller Krankheiten seines Versicherten bereits im voraus bekannt wären, so würde er eine Prämie verlangen, die dem Barwert der zukünftigen Leistungen entspricht bzw. diesen übersteigt. Mit der Anlage dieses Geldes auf dem Finanzmarkt könnte der Versicherte dann ebensogut seine Krankheitskosten finanzieren.

Risikodiskriminierung kann zudem das Gerechtigkeitsempfinden verletzen, wenn sie an Merkmalen ansetzt, für die der Betreffende gar nicht verantwortlich ist. Dies ist bei Geschlecht, Alter und Vorerkrankungen der Fall. Eine noch weitergehendere Risikodifferenzierung, etwa mit dem Instrument der Gen-Analyse, dessen Einsatz bereits gefordert wird, verstärkt die Diskriminierung und wird vielfach aus ethischen Gründen abgelehnt. ${ }^{15}$ Demgegenüber kommt eine Risikodifferenzierung Gerechtigkeitsvorstellungen näher, wenn sie an Merkmalen ansetzt, für die der Versicherte verantwortlich ist. Zu denken wäre beispielsweise an Tabakkonsum oder Körpergewicht.

Inwieweit Risikodiskriminierung praktiziert wird, ist eine Frage der Versicherungsgestaltung. In der gesetzlichen Krankenversicherung wird bewußt auf jede Risikodiskriminierung verzichtet. Dieser Leistungsrisikoausgleich wird von uns als versicherungsimmanente Umverteilung

\footnotetext{
14 Vgl. Henke, K.-D./ Behrens, C., 1989; Andel, N., 1975; Ott, G. 1981.
}

15 Vgl. Hannoversche Allgemeine Zeitung vom 20.2.1997, S.8. 
interpretiert. Dementsprechend läßt sich eine äquivalente Prämie berechnen, die für jeden Versicherten gilt, unabhängig von Vorerkrankungen, Alter oder Geschlecht. Diese Praxis der gesetzlichen Krankenversicherung spart Verwaltungskosten. ${ }^{16}$ Sie stellt auch verfassungsrechtlich kein Problem dar: "In der Funktion einer Versicherungsprämie ist der Krankenversicherungsbeitrag stets verfassungsgerecht. Daran ändert die Eigentümlichkeit der Sozialversicherung nichts, die Versicherungsprämie im Gegensatz zur Privatversicherung nach dem Gesamtrisiko aller Versicherten statt nach dem individuellen Risiko des jeweiligen Versicherten zu bemessen". ${ }^{17}$

Da in der GKV der Beitrag nach einem Durchschnittsversicherten berechnet wird, erfolgt als eine Form des Leistungsrisikoausgleichs ein Altersausgleich. Die "nach Art der Lebensversicherung" kalkulierten privaten Krankenversicherungen müssen dagegen, wenn sie heute die Prämie für einen 20jährigen Versicherungsnehmer ermitteln wollen, dessen Krankheitskosten im Alter von 80 Jahren abdiskontiert in die Kalkulation einbeziehen. Es handelt sich dabei um die Krankheitskosten eines 80jährigen im Jahre 2058. Da diese kaum abzuschätzen sind, werden vereinfachend die Krankheitskosten eines 80jährigen zum Zeitpunkt der Kalkulation zugrundegelegt, evtl. versehen mit einem Sicherheitsaufschlag. Erweist sich die auf solcher Grundlage gebildete Alterungsrïckstellung als zu niedrig, werden Beitragsanpassungen notwendig. Auch GKV-Mitglieder unterliegen Beitragsanpassungen infolge steigender Krankheitskosten. Doch werden sie gegen die höheren Krankheitskosten im Alter statt durch eine Altersrückstellung durch den Leistungsrisikoausgleich geschützt. Die Last von über die gesamte Vertragsdauer zu geringen Zuführungen zu einer Altersrückstellung kann sie nicht treffen.

Bei der im folgenden vorzunehmenden Berechnung des äquivalenten Beitrags nach Leistungsrisikoausgleich werden zunächst die quantitativ wenig relevanten beitragsabhängigen Leistungen (Krankengeld) betrachtet. Erst im zweiten Schritt wird der äquivalente Beitrag für die das Gros der GKV-Leistungen ausmachenden beitragsunabhängigen Leistungen ermittelt, denn dies ergibt sich durch eine bloße Vereinfachung der Betrachtung zu Ersterem. Am Ende werden beide Ergebnisse zu einer Funktion zusammengefügt.

\footnotetext{
16 Vgl. Kleinhenz, G.D., 1992, S.62.
}

17 Kirchhof, F., 1994, S.1324 f. 


\subsection{2 Äquivalenz bei beitragsabhängigen Leistungen}

Die gesetzliche Krankenversicherung gewährt ihren Versicherten eine Vielzahl von Leistungen, von der Gesundheitsförderung und Krankheitsverhütung über Krankenbehandlung und Krankengeld bis hin zu Leistungen bei Schwangerschaft und Mutterschaft. ${ }^{18}$ Nun muß unterschieden werden, ob der Wert dieser Leistungen von der Höhe des Beitrags abhängig oder unabhängig ist. Ein direkter Zusammenhang zwischen Beitragshöhe und Wert der Leistung besteht beim Krankengeld und beim Mutterschaftsgeld. Das Mutterschaftsgeld wird hier wegen geringer quantitativer Bedeutung und teilweiser Finanzierung durch eine Erstattung des Bundes ausgeklammert. Wir gehen im folgenden von einem Krankengeld in Höhe von 80 v.H. der jeweiligen Beitragsbemessungsgrundlage (im folgenden BBMG), meist also des erzielten regelmäßigen Arbeitseinkommens bis zur Beitragsbemessungsgrenze, aus. ${ }^{19}$

Betrachtet wird nun eine Gesamtheit von L in der GKV versicherten Personen, die alle vom Risiko eines krankheitsbedingten Einkommensausfalls bedroht sind. Ein Versicherter i $(\mathrm{i}=1, \ldots, \mathrm{L})$ wird innerhalb einer Betrachtungsperiode $\mathrm{m}_{\mathrm{i}}-\mathrm{mal}\left(0 \leq \mathrm{m}_{\mathrm{i}} \leq \infty\right)$ vom Zufallsereignis Leistungsfall, d.h. von einer einen Krankengeldanspruch begründenden Krankheit, betroffen. Die Dauer der Krankheit beträgt jeweils $d_{i, j}\left(j=0, . ., m_{i}\right)$ Tage. Insgesamt ist der Versicherte i in der Betrachtungsperiode

$$
d_{i}=\sum_{j=0}^{m_{i}} d_{i, j}
$$

Tage krank. Die Gesamtzahl von Krankheitstagen im Kollektiv L beträgt

$$
\mathrm{D}=\sum_{\mathrm{i}=1}^{\mathrm{L}} \mathrm{d}_{\mathrm{i}}
$$

Der Krankengeldanspruch eines Versicherten i $\left(\mathrm{KG}_{\mathrm{i}}\right)$ beläuft sich dementsprechend auf

18 Eine Übersicht über die Leistungen enthält $§ 21$ Abs. 1 SGB I.

19 Dies entspricht dem Rechtsstand vor dem 1.1.1997. Durch Art. 2 Nr. 14 des Gesetzes zur Entlastung der Beitragssätze in der gesetzlichen Krankenversicherung (Beitragsentlastungsgesetz - BeitrEntlG) vom 1. November 1996 (BGBl I S. 1631) wird mit Wirkung ab 1. Januar 1997 die Höhe des Krankengeldes von bisher 80 auf 70 vom Hundert des Regelentgelts herabgesetzt. Da sich unsere Modellrechnungen jedoch durchweg auf Zeitpunkte vor 1997 beziehen, wird diese Änderung nicht einbezogen. 


$$
\mathrm{KG}_{\mathrm{i}}=\mathrm{d}_{\mathrm{i}} \times 0,8 \times \mathrm{BBMG} / \mathrm{Tag} .
$$

Da $d_{i}$ eine Zufallsvariable ist, ist ex ante nicht bekannt, wie hoch der Krankengeldanspruch eines bestimmten Versicherten sein wird. Um den Wert einer Krankengeldversicherung eines Versicherten zu bestimmen, muß deshalb der Erwartungswert von Krankheitstagen (E(d)) zugrundegelegt werden. Diesen kann man mit der Schätzfunktion

$$
\hat{\mathrm{E}}(\mathrm{d})=\overline{\mathrm{d}}=\mathrm{D} / \mathrm{L}
$$

ermitteln. Die Schätzung durch das arithmetische Mittel ist dann erwartungstreu, wenn man davon ausgeht, daß alle Versicherten den gleichen Erwartungswert an Krankheitstagen besitzen. Da keine Risikodifferenzierungen vorgenommen, mit anderen Worten die einzelnen Versicherten nicht in Risikoklassen eingeordnet werden, ist die Berechnung des Erwartungswertes nach dem Durchschnittsrisiko folgerichtig. Der Wert der Krankengeldversicherung $\left(\mathrm{W}^{\mathrm{KGV}}\right)$ beträgt dann

$$
\mathrm{W}^{\mathrm{KGV}}=\hat{\mathrm{E}}(\mathrm{d}) \times 0,8 \times \mathrm{BBMG} / \mathrm{Tag} .
$$

Einzig die BBMG kann zwischen Versicherten variieren, so daß $\mathrm{W}^{\mathrm{KGV}}=\mathrm{f}(\mathrm{BBMG})$ gilt. Es handelt sich um eine lineare Funktion, die mit $\mathrm{BS}=\hat{\mathrm{E}}(\mathrm{d}) \times 0,8$ (BS ist ein konstanter Beitragssatz) als $\mathrm{W}^{\mathrm{KGV}}=\mathrm{BS} \times \mathrm{BBMG}$ geschrieben werden kann. Es muß ein fixer Beitragssatz auf die BBMG erhoben werden, um zu einem äquivalenten Beitrag zu kommen.

Vor diesem Hintergrund kann beispielhaft ermittelt werden, welcher Teil des GKVBeitragssatzes im Jahr 1995 durch den Wert der Krankengeldversicherung gerechtfertigt war. Dabei wird vereinfachend davon ausgegangen, daß alle GKV-Mitglieder ein einziges großes Versichertenkollektiv bilden. Zwar gibt es "die" GKV nicht, sondern nur $607^{20}$ einzelne gesetzliche Krankenkassen, jedoch findet zwischen den einzelnen Kassen ein Risikostrukturausgleich statt, der die Annahme eines einzigen Versichertenkollektivs vertretbar werden läßt. Des weiteren wird angenommen, alle GKV-Mitglieder seien in gleicher Weise krankengeldberechtigt, beitragsfrei Mitversicherte jedoch nicht. Dies ist näherungsweise richtig. Genau genommen gibt es unterschiedliche bei den Mitgliedern Krankengeldberechtigungen (sofortige Berechtigung,

20 Stand 31.12.1996. Angaben des Bundesministeriums für Gesundheit. 
nach Ablauf des Entgeltfortzahlungsanspruchs, kein Anspruch) und entsprechend erhöhte ( $\S$ 242 SGB V) oder ermäßigte ( $\$ 243$ SGB V) Beitragssätze. Auch hängt die Anspruchsberechtigung der freiwilligen Mitglieder vom Satzungsrecht der jeweiligen Krankenkasse ab.

Schließlich wird - von der Belastungsinzidenz her unproblematisch - keine Unterscheidung in Arbeitnehmer- und Arbeitgeberbeitrag vorgenommen.

Bekannt sind die summierten Beitragsbemessungsgrundlagen ( $\Sigma B B M G=1.400 \mathrm{Mrd}$. DM) sowie die Summe des ausgezahlten Krankengeldes $(\Sigma \mathrm{KG}=18,4 \mathrm{Mrd} . \mathrm{DM}){ }^{22}$ Da wir von einem einheitlichen Beitragssatz ausgehen, muß gelten: $\Sigma \mathrm{W}^{\mathrm{KGV}}=\mathrm{BS} \times \Sigma \mathrm{BBMG}$. Da $\mathrm{W}^{\mathrm{KGV}}$ eine erwartungstreue Schätzung für den tatsächlichen Krankengeldanspruch ist, gilt für das Versichertenkollektiv $\Sigma \mathrm{W}^{\mathrm{KGV}}=\Sigma \mathrm{KG}$ und $\Sigma \mathrm{KG}=\mathrm{BS} \times \Sigma \mathrm{BBMG}$. Daraus ergibt sich für einen Durchschnittsversicherten in der Versicherungsperiode 1995 ein durch die Krankengeldversicherung gerechtfertigter Beitragssatz von 1,31 v.H. Der jahresdurchschnittliche allgemeine Beitragssatz in der GKV lag im Jahr 1995 jedoch bei 13,2 v.H. (West) und 12,8 v.H. (Ost). Mit der Differenz wurden die vielen anderen, von der Beitragshöhe unabhängigen Leistungen der GKV finanziert, insbesondere die Krankenbehandlung und auch die Verwaltung.

\subsection{3 Äquivalenz bei beitragsunabhängigen Leistungen}

Sowohl die Gewährung als auch die Höhe der beitragsunabhängigen Leistungen richtet sich nach dem Bedarfsprinzip. Eine Differenzierung der Beiträge proportional zum Arbeitsentgelt kann für diesen Teil des Versicherungsschutzes kein Weg zur Beitragsäquivalenz sein. Es stellt sich damit die Frage nach dem äquivalenten Beitrag für diesen Teil der Versicherungsleistungen.

Es wird erneut ein Versicherter i $(i=1, . ., \mathrm{L})$ aus dem Versichertenkollektiv L betrachtet, der innerhalb der Betrachtungsperiode $\mathrm{n}_{\mathrm{i}}$-mal (mit $0 \leq \mathrm{n}_{\mathrm{i}} \leq \infty$ ) von einem anspruchsbegründenden Versicherungsfall betroffen wird. Jeder Versicherungsfall $\mathrm{k}$ löst die Versicherungsleistung $\mathrm{v}_{\mathrm{i}, \mathrm{k}}$ $\left(\mathrm{k}=0, . ., \mathrm{n}_{\mathrm{i}}\right)$ aus. Insgesamt bekommt der Versicherte i die Leistungen

21 Stand 1.1.1996. Angaben des Bundesministeriums für Gesundheit, Referat 228.

22 Angaben des Bundesministeriums für Gesundheit, Referat 228 (Schätzwerte). 


$$
\mathrm{v}_{\mathrm{i}}=\sum_{\mathrm{k}=0}^{\mathrm{n}_{\mathrm{i}}} \mathrm{v}_{\mathrm{i}, \mathrm{k}}
$$

Die Gesamtleistungen der gesetzlichen Krankenversicherung betragen dann

$$
\mathrm{V}=\sum_{\mathrm{i}=1}^{\mathrm{L}} \mathrm{v}_{\mathrm{i}}
$$

Um den Wert einer Versicherung dieser Leistungen zu bestimmen, muß wieder ihr Erwartungswert (E(v)) zugrundegelegt werden. Diesen kann man im Kollektiv L erwartungstreu und effizient mit der Schätzfunktion $\hat{E}(v)=V / L$ ermitteln. Wie der Ausdruck zeigt, ist der Wert des Versicherungsschutzes für diese Leistungen unabhängig von geleisteten Beiträgen und konstant.

Der äquivalente Beitrag für das Jahr 1995 kann, von den Annahmen der vorangehenden Rechnung ausgehend, bestimmt werden. Im Gegensatz zum Krankengeld, welches nur Mitgliedern zusteht, müssen hier alle Versicherten, d. h. auch die beitragsfrei Mitversicherten, einbezogen werden. Im Jahr 1995 erreichten die Gesamtausgaben der gesetzlichen Krankenversicherung 259,7 Mrd. DM. ${ }^{23}$ Davon waren 18,4 Mrd. DM Krankengeld, so daß auf die übrigen Leistungen 241,3 Mrd. DM verwendet wurden. Die Zahl der Versicherten belief sich in diesem Jahr auf 72,154 Mill. $^{24}$ Es ergibt sich, auf das Jahr und einen Versicherten bezogen, ein Erwartungswert beitragsunabhängiger Leistungen von 3344,24 DM. Das entspricht einem äquivalenten monatlichen Beitrag von 278,67 DM. Da von den Gesamtausgaben und nicht nur von den gesamten Leistungsausgaben ausgegangen wurde, wurden implizit die Verwaltungskosten gleichmäßig auf die einzelnen Versicherungsverhältnisse verteilt.

\subsection{4 Äquivalenz für den Gesamtversicherungsschutz}

Der äquivalente Beitrag $\left(\mathrm{B}^{\mathrm{a}}\right)$ für den Gesamtversicherungsschutz durch die GKV läßt sich für das Jahr 1995 durch folgenden funktionalen Zusammenhang darstellen:

$$
B^{\ddot{a}}=f(B B M G)=0,0131 \times B B M G+278,67
$$

\footnotetext{
23 Angaben des Bundesministeriums für Gesundheit, Referat 228.

24 Eigene Berechnungen nach: Statistisches Bundesamt, 1996, S.11.
} 
Die Darstellung der äquivalenten Beitragsfunktion in Kontrast zur 1995 gültigen durchschnittlichen Beitragsfunktion ergibt ein Bild von den derzeitigen Über- und Unterbelastungen der GKV-Mitglieder.

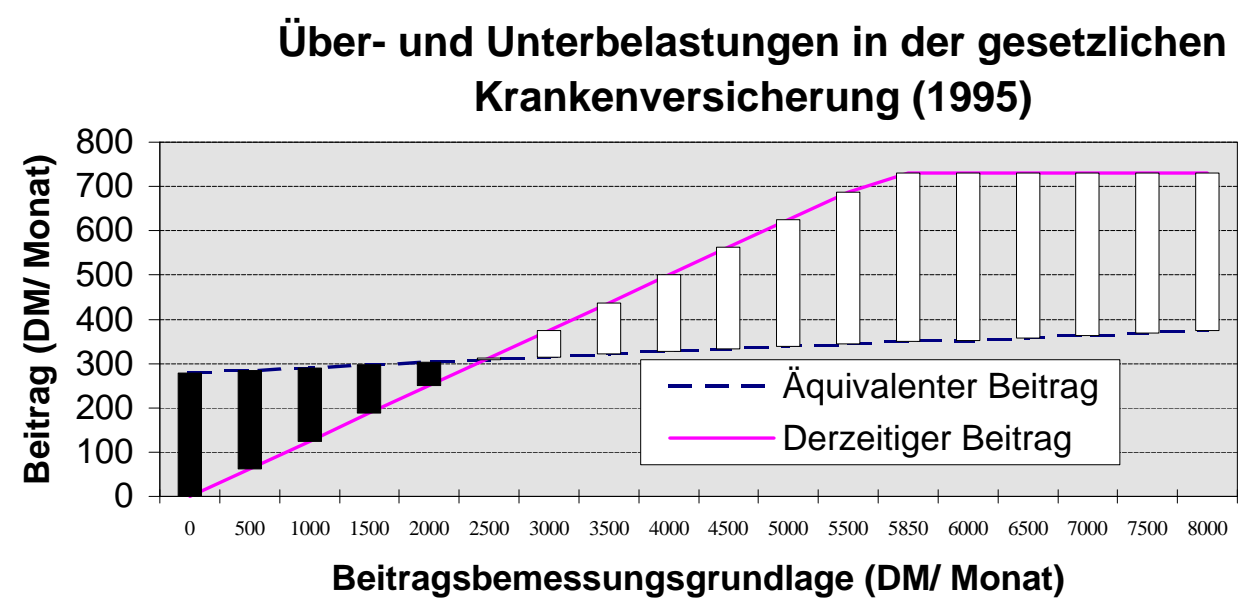

Es ist bemerkenswert, wie niedrig der äquivalente Beitrag im Verhältnis zum tatsächlichen Beitrag ist. Dies hängt stark damit zusammen, daß ihn alle GKV-Versicherten entrichten müßten. Die beitragsfreie Versicherung, die im Jahre 1995 22,6 Mill. Versicherte auf Kosten der GKV-Mitglieder genossen, gibt es in dieser Kalkulation natürlich nicht. Bemerkenswert auch, daß ein GKV-Mitglied schon bei einem beitragspflichtigen Einkommen von ca. 2470 DM/Monat (1995) in die Zone der Überbelastungen wechselt, und beginnt, den sozialen Ausgleich mitzufinanzieren.

\subsection{Die Apologie des sozialen Ausgleichs}

Wie gezeigt wurde, führt die derzeitige Praxis der Beitragsberechnung zu Über- und Unterbelastungen einzelner Versichertengruppen. Dies steht in einem eindeutigen Konflikt mit dem Grundsatz der Belastungsgleichheit. Während die Unterbelastungen möglicherweise aus sozialpolitischen Gründen erwünscht sind (s.u. Kapitel 3), stellen die Überbelastungen einen ungerechtfertigten Teil einer Sonderlast dar. Die betroffene Personengruppe müßte hiergegen den Schutz des Grundsatzes der Belastungsgleichheit in Anspruch nehmen können. Immerhin konnte diese Überbelastung für ein GKV-Mitglied im Jahr 1995 bis zu 4580 DM betragen. ${ }^{25}$

25 Äquivalenter Beitrag, subtrahiert vom derzeitigen Beitrag hochgerechnet auf das ganze Jahr. Fälle oberhalb der Beitragsbemessungsgrenze. 
Zur Rechtfertigung dieser Sonderbelastung wird von verfassungsrechtlicher Seite ins Feld geführt, das Prinzip des sozialen Ausgleichs sei durch die Erwähnung der Sozialversicherung in den Art. 74 und 87 vom Grundgesetz anerkannt worden. ${ }^{26}$ Gleichzeitig wird betont, daß der soziale Ausgleich nur in den "traditionellen Grenzen und Formen trotz der Bedenken aus dem Gleichheitssatz gebilligt" ${ }^{27}$ sei.

Hierzu ist anzumerken, daß zur Zeit der Konzeption der wesentlichen Strukturmerkmale der GKV zum Ende des letzten Jahrhunderts die proportional vom Arbeitsentgelt abhängigen Leistungen dominierten. Vorrangige Funktion der GKV war damals, den krankheitsbedingten Einkommensausfall, der die Arbeiterfamilien schnell in Existenznot bringen konnte, abzuwehren. Die medizinischen Leistungen traten aufgrund der geringen Therapiemöglichkeiten in den Hintergrund. So entfielen damals knapp 60 v.H. der Ausgaben auf Einkommensersatzleistungen $^{28}$, während es im Jahr 1996 nur noch 6,8 v.H. waren. Und sogar die medizinischen Leistungen wurden damals teilweise über arbeitsentgeltabhängige Kostenerstattungen gewährt. So sah $\S 75$ des Krankenversicherungsgesetzes von 1883 vor, daß anstelle der freien ärztlichen Behandlung eine Erhöhung des Krankengeldes um ein Viertel des "üblichen Lohns" gewährt werden konnte. ${ }^{29}$ Dies war unproblematisch, weil das Entgelt für Arzt und Arzneien aufgrund fehlender medizinisch-technischer und pharmazeutischer Möglichkeiten gering und kalkulierbar war.

Damit führte die arbeitsentgeltabhängige Bemessung der Beiträge eher zu einer Beitragsäquivalenz als zu einen sozialen Ausgleich. Auch konnte damals von einer recht einheitlichen Familienstruktur und einem recht einheitlichen Familenlebenszyklus ausgegangen werden, so daß auch die beitragsfreie Mitversicherung von Familienangehörigen kein großes Ausgleichselement beinhaltete.

Erst mit den zunehmenden medizinischen Leistungsmöglichkeiten bei gleichzeitiger Reduzierung der Einkommensersatzfunktionen, z.B. durch die gesetzliche Einführung des 6-wöchigen Lohnfortzahlungsanspruchs für Arbeiter im Jahr 1967, trat die Beitragsäquivalenz zugunsten

\footnotetext{
26 Vgl. z.B. Kirchhof, F., 1994, S.1327. Es stellt sich dem verfassungsrechtlichen Laien die Frage, weshalb die bloße Erwähnung einer Institution im Grundgesetz einer durch die Verfassung bescheinigten Unbedenklichkeit gleichkommt. Eine solche Rechtfertigung müßte dann z.B. auch für die Einkommensteuer gelten, der man "im Nachhinein" jedoch verfassungsrechtliche Mängel nachgewiesen hat.

27 Ebd. S.1327 mit weiteren Nachweisen auch von BVerfG-Urteilen.

28 Vgl. z.B. Wasem, J., 1994, S.111.
} 
des sozialen Ausgleichs in den Hintergrund. Und die finanziell erheblichen Dimensionen des sozialen Ausgleichs in Folge von steigenden Beitragssätzen und Beitragsbemessungsgrenzen sind ein Phänomen der jüngsten Geschichte. So hat der Durchschnittsbeitragssatz Ende 1885 nur etwa 1,8 v.H. ${ }^{30}$ und im Jahre 1970 etwas über 8 v.H. betragen, während heute über 13 v.H. des Arbeitsentgelts bis zur Beitragsbemessungsgrenze in der Krankenversicherung verwendet und dem sozialen Ausgleich unterzogen wird. Von der Wahrung "traditioneller Grenzen und Formen" kann somit insbesondere in quantitativer Hinsicht nicht gesprochen werden.

Ein weiterer Rechtfertigungsversuch für den sozialen Ausgleich könnte, Männer folgend ${ }^{31}$, auf der Interpretation des sozialen Ausgleichs als Versicherung gegen das Risiko, infolge einer zukünftigen Einkommensänderung den äquivalenten Beitrag nicht mehr entrichten zu können, beruhen. Eigenschaft einer derartigen Versicherung wäre, daß sie im Fall einer „NichtBezahlbarkeit von Beiträgen“332, ebendiese subventioniert, im Fall ausreichender Leistungsfähigkeit aber eine zusätzliche Prämie für diese Leistung erhebt. Die Beitragshöhe in der gesetzlichen Krankenversicherung steht jedoch, wie gleich gezeigt wird (GP 3), in keinem engen Verhältnis zur Leistungsfähigkeit oder sozialen Bedürftigkeit eines Versichertenhaushaltes. Somit wirkt der soziale Ausgleich nicht oder nur sehr unvollkommen als Versicherung gegen die „Nicht-Bezahlbarkeit“" von Beiträgen.

Ein weiterer Ansatzpunkt zur Begründung des sozialen Ausgleichs hängt mit dem Begriff der Gruppenhomogenität zusammen. ${ }^{33}$ Es wird argumentiert, daß Mitglieder einer Gruppe eine besondere Verantwortung oder Solidarität untereinander hätten, die die Überbelastung bestimmter Gruppenmitglieder zugunsten anderer rechtfertige. Hier ist zusätzlich zu dem vorstehend angeführten Bedenken festzuhalten, daß es sich um eine staatlich verordnete Solidarität unter Mitgliedern einer mit staatlichem Zwang zusammengeführten Gruppe handelt. Dieses Konstrukt könnte zwar dazu dienen, ein tatsächlich vorhandenes Solidaritätsempfinden nachzuzeichnen. Es nützt allerdings sowohl traditionell als auch tatsächlich erheblich den Nichtmitgliedern der Solidargemeinschaft, die hierdurch von Verantwortung und Finanzierungspflichten frei werden. Insbesondere, soweit es sich bei den Unterbelastungen in der gesetzlichen Kran-

29 Vgl. Schulin, B., 1994, S.197.

30 Vgl. Schlenker, R.-U., 1994, S.8.

31 Vgl. Männer, L., 1997, S. 217 ff. und 224.

32 Ebd. 
kenversicherung um Beiträge zu allgemeinen Staatszielen ${ }^{34}$ handelt, werden gesamtstaatliche Aufgaben von einer Sonderheit von Bürgern finanziert. Hierfür gibt es auch sehr eindeutige Beispiele: So bezieht $\S 5$ Abs. 1 Nr. 5 bis 8 SGB V soziale Problemgruppen (Personen in Einrichtungen der Jugendhilfe, Rehabilitanden und Behinderte) in die GKV ein, die größtenteils auf der Empfängerseite stehen werden. Auch die stark reduzierten Beiträge für Studenten und Praktikanten wären hier zu nennen. Die verpflichtende Bildung einer Solidargemeinschaft und zwangsweise Einbeziehung von Empfängergruppen ermöglicht es, die Nichtmitglieder der Solidargemeinschaft aus sozialstaatlichen Verantwortlichkeiten zu entlassen.

Mit Einführung des Risikostrukturausgleichs durch das Gesundheitsstrukturgesetz im Jahre 1993 sind überdies die solidargemeinschaftlichen Grenzen weitgehend aufgehoben worden. Durch den Risikostrukturausgleich und die Wahlfreiheit der Versicherten hinsichtlich der Versicherung sind die einzelnen Risikogemeinschaften, die sich teilweise noch anhand bestimmter z.B. berufsständischer, regionaler oder anderer Merkmale abgegrenzt haben, quasi zu einer großen Versicherungsgemeinschaft verschmolzen worden. Die Risikostrukturen werden finanziell ausgeglichen, so daß, um einmal ein Beispiel zu bilden, die überregionale Risikogemeinschaft der "Techniker-Krankenkasse", die vornehmlich Versicherte aus technischen Berufen umfaßt, für die durch überdurchschnittlich viele Arbeitslose und chronisch Kranke geschwächte regionale Risikogemeinschaft "Allgemeine Ortskrankenkasse Berlin" finanziell einstehen muß. Wollte man also die Argumentation mit der besonderen "Gruppenhomogenität" aufrechterhalten, so müßte diese nun für das Gesamtkollektiv der gesetzlich Krankenversicherten geführt werden.

\section{Sozialpolitische Effizienz des sozialen Ausgleichs}

Die Tatsache, daß die Beitragssubventionen auf das Wirken der aus dem vorigen Jahrhundert zumeist unverändert übernommenen Strukturelemente der gesetzlichen Krankenversicherung ${ }^{35}$ im zeitgenössischen sozioökonomischem Umfeld zurückzuführen sind, läßt vermuten, daß schon die sozialpolitische Effektivität dieser Leistungen gering ist. Zudem dürften hohe "Streu-

33 Vgl. Schulin, B., 1994, S.233, m. w. N.

$34 \mathrm{Zu}$ denken wäre hier beispielsweise an die Förderung der Familie und der Ehe nach Art. 6 GG oder an die Sicherung des gemäß Art 1f. Grundgesetz jedem Bürger zu gewährenden Existenzminimums.

35 Vgl. hierzu auch Gliederungspunkt 2.2. 
verluste" auftreten, d.h. auch die sozialpolitische Effizienz dürfte gering sein. ${ }^{36}$ Beides läßt sich wissenschaftlich nur bedingt überprüfen, weil die sozialpolitischen Ziele, die mit dem sozialen Ausgleich erreicht werden sollen, von einem demokratischen Gesetzgeber noch nie ausformuliert wurden. ${ }^{37}$ Wenn jedoch gefragt wird, welchen allgemeinen sozialpolitischen Zielen der soziale Ausgleich dient, so kommen eigentlich nur zwei in Frage: Die arbeitsentgeltabhängige proportionale Beitragsbemessung entlastet einkommenschwache Haushalte, während die beitragsfreie Mitversicherung von Familienangehörigen insbesondere Haushalte mit Kindern begünstigt.

Im Gegensatz zum Einkommensteuerrecht fällt es schwer, den expliziten Willen einer Unterstützung auch kinderloser Ehen zu erkennen. So kann ein Ehepartner eines Pflichtversicherten nur beitragsfrei mitversichert werden, wenn sein Einkommen die Grenze des $\S 10$ Abs. 1 Nr. 5 SGB V unterschreitet. Dies ist in der Regel dann der Fall, wenn der Ehegatte aus dem Erwerbsleben ausscheidet, um sich der Kinderbetreuung zu widmen. Liegt der Verzicht auf eine eigene Erwerbstätigkeit in dem Einkommen des Partners begründet, ist ebenfalls eine beitragsfreie Mitversicherung möglich. Dies dürfte aber eher toleriert denn intendiert sein. Somit ist vorläufig die Zielgruppe mit als "einkommenschwache Haushalte unter besonderer Berücksichtigung von Kindern" eingegrenzt. Der Begriff der "Einkommensschwäche" bedarf einer genaueren Betrachtung.

\subsection{Das Netto-Äquivalenzeinkommen}

Darüber, ob ein Mitglied Nettoempfänger oder Nettozahler im sozialen Ausgleich der gesetzlichen Krankenversicherung wird, entscheidet derzeit im wesentlichen nur ein Indikator, nämlich die Höhe des Arbeitsentgelts. Dies war zur Zeit der Entstehung der gesetzlichen Krankenversicherung im vorigen Jahrhundert zeitgerecht. Die damalige Zielgruppe der Arbeiter verfügte in der Regel nur über das Arbeitsentgelt als Einkunftsquelle. Andere Familienmitglieder verfügten entweder über kein eigenes Einkommen (insbesondere Heranwachsende und nicht berufstätige Ehefrauen) oder standen selbst in einem Arbeitsverhältnis. Im ersten Fall erschien ihre bei-

\footnotetext{
36 Zu Grundfragen der Zielgenauigkeit von Sozialleistungen vgl.: Atkinson, A. B. , 1994, S.(3-1)-(3-8).

37 Vgl. Wasem, J., 1994, S.99. ZU der Problematik nicht konkretisierter politischer Zielvorstellungen vgl. insbesondere: Atkinson, A.B., 1994, S.(3-8) u. Lampert, H., 1995, S.521, 524.

38 Die beitragsfreie Mitversicherung bei freiwilliger Versicherung ist satzungsabhängig geregelt.
} 
tragsfreie Mitversicherung sinnvoll, im zweiten Fall waren sie selbst beitragspflichtig versichert.

Die sozioökonomischen Strukturen im heutigen Versichertenkollektiv sind vielfältiger. Der Anteil der Einkünfte aus Kapitalvermögen, aus selbständiger Tätigkeit sowie aus Vermietung und Verpachtung ist bei den gesetzlich Krankenversicherten erheblich. Das Arbeitsentgelt allein ist deshalb kein geeigneter Indikator mehr für Bedürftigkeit oder Leistungsfähigkeit in einem sozialen Ausgleich.

Ein Meßkonzept, das die Einkommenssituation aller GKV-Versicherten trotz heterogener Haushalts- und Einkommensstrukturen auf einen Nenner bringen kann, ist das NettoÄquivalenzeinkommen. Dieses Konzept trägt dem Umstand Rechnung, daß mit einem Einkommen oft der Lebensunterhalt mehrerer Haushaltsmitglieder bestritten wird und berücksichtigt gleichzeitig, daß Mehrpersonenhaushalte günstiger wirtschaften können als Einpersonenhaushalte. Es unterscheidet sich vom pro-Kopf Haushaltseinkommen dadurch, daß nicht alle Haushaltsmitglieder mit dem Wert eins, sondern unterschiedlich gewichtet werden. Hierfür steht eine große Zahl von Äquivalenzskalen zur Wahl. ${ }^{39}$ Die empirische Sozialforschung in Deutschland verwendet häufig eine aus den Regelsatzproportionen der Sozialhilfe abgeleitete Skala. Diese ist im internationalen Vergleich, etwa zur amerikanischen Skala, zur OECD Skala wie auch zu der zuletzt von Eurostat verwendeten Skala sehr kinderfreundlich. Die "deutsche Bedarfsgewichtung" ${ }^{40}$ unterstellt geringere Vorteile gemeinsamen Wirtschaftens und weist Kindern ein relativ höheres Gewicht zu. Wird beispielsweise ein Haushaltsmitglied unter 14 Jahren nach der OECD-Skala pauschal mit 50\% des Grundbedarfs eines erwachsenen Haushaltsvorstandes angesetzt, differenziert das deutsche System stärker nach dem Alter der Kinder, deren Gewichte zwischen $45 \%$ und $90 \%$ erreichen. Auch weitere Erwachsene werden in größerem Maß berücksichtigt ( $80 \%$ gegenüber $70 \%$ in der OECD-Skala). Dementsprechend ist das Äquivalenzeinkommen von Personen, die in größeren Haushalten oder in Haushalten mit Kindern leben, bei Nutzung der "deutschen Skala" relativ gering. ${ }^{41}$ In der Konsequenz wird

39 Buhmann et al. stellen alleine 30 verschiedene Skalen, die einen Effekt der Haushaltsgröße auf die Bedarfssituation und Bedarfsdeckungsmöglichkeiten berücksichtigen, zusammen. Vgl. Buhmann, B. et al., 1988, S.116 u. S.119 ff. Vgl. auch Bundestags-Drucksache 13/3339, 1995, S.7.

40 Es handelt sich dabei nicht um eine offizielle Skala, sondern um eine den politisch-administrativen Regelungen nachgebildete Bedarfsgewichtung, die mit Buhmann et al. (1988, S.121) auch als implizite Expertenskala bezeichnet werden kann. In der Bundesrepublik wird von offizieller Seite weder mit dem NettoÄquivalenzeinkommen gearbeitet noch eine darauf bezogene offizielle Armutsgrenze gesetzt. 
für diese Haushalte ein höherer Ausgleichsbedarf signalisiert als dies bei Nutzung der genannten anderen Gewichtungssysteme der Fall wäre.

In der folgenden empirischen Analyse wird ganz bewußt eine Äquivalenzgewichtung vorgenommen, die auf den im Betrachtungsjahr gültigen Regelsatzproportionen der Sozialhilfe basiert. Damit wird dem hohen Rang entsprochen, den die Familienförderung im Zielsystem der deutschen Sozialpolitik sowie im sozialen Ausgleich der gesetzlichen Krankenversicherung einnimmt. Die Untersuchung bezieht sich auf das Jahr 1989, da für dieses Jahr letztmalig Angaben über Beiträge zur gesetzlichen Krankenversicherung im hier verwendeten SOEPDatensatz zu Verfügung stehen.

\subsection{Die Nettoposition als Indikator für die Stellung im sozialen Ausgleich}

Als Indikator für Begünstigung und Belastung im sozialen Ausgleich kann aufbauend auf Überlegungen in Gliederungspunkt 2.3 eine "Nettoposition" bestimmt werden. Die Nettoposition eines Versicherten i ergibt sich aus dem äquivalenten Beitrag $\mathrm{B}^{\ddot{a}}$ abzüglich des tatsächlichen Beitrags $\mathrm{B}^{\mathrm{t}}$.

$$
\mathrm{NP}_{\mathrm{i}}^{\mathrm{VS}}=\mathrm{B}^{\ddot{\mathrm{a}}-\mathrm{B}^{\mathrm{t}}}
$$

Ist die Nettoposition negativ, bedeutet dies, daß der Versicherte ein Zahler im sozialen Ausgleich ist. Die Nettoposition eines Haushalts $\mathrm{j}$, in dem die Versicherten $\mathrm{VS}_{1}, . . \mathrm{VS}_{\mathrm{s}}$ leben, ergibt sich aus:

$$
\mathrm{NP}_{\mathrm{j}}^{\mathrm{HH}}=\sum_{\mathrm{r}=1}^{\mathrm{s}} \mathrm{NP}_{\mathrm{r}}^{\mathrm{V}}
$$

Dabei können in einem solchen Haushalt auch Personen leben, die privat oder gar nicht krankenversichert sind. Sie bleiben bei der Berechnung der Nettoposition des Haushalts außer Acht.

41 Vgl. Hauser, R., 1995, S.4.; Burkhauser, R.V. et al, 1994, S.153 f. u. S.159, Buhmann, B. et al., 1988, S.119, 121, 135 . 
Als tatsächlicher Beitrag $B^{t}$ wird im folgenden der Gesamtbeitrag inklusive des sogenannten Arbeitgeberbeitrags verstanden. ${ }^{42}$ Gäbe es den Arbeitgeberbeitrag nicht und wäre der Arbeitnehmerbeitrag entsprechend höher, so stünde zu erwarten, daß es über den Verhandlungsmechanismus zu höheren Löhnen und Gehältern käme. Unter neoklassischen Bedingungen würde diese Erhöhung genau der Erhöhung des Arbeitnehmerbeitrags entsprechen. ${ }^{43}$ Der Arbeitgeberbeitrag wird deshalb hier analog zur steuerrechtlichen Betrachtungsweise als Lohnbestandteil, der zur Beitragszahlung verwendet wird, behandelt.

Zur Berechnung des äquivalenten Beitrages sind erneut die Gesamtausgaben der gesetzlichen Krankenversicherung und die Versichertenzahl heranzuziehen. Die Gesamtausgaben bezifferten sich 1989 auf 129,9 Mrd. DM ${ }^{44}$. Die Zahl der Versicherten einschließlich beitragsfrei Mitversicherter wurde auf Grundlage des Sozio-ökonomischen Panel ermittelt und beläuft sich auf 56,6 Mill. Vernachlässigt wird im folgenden die geringfügige Abhängigkeit des äquivalenten Beitrags vom Arbeitsentgelt (vgl. Gliederungspunkt 2.3.2). Vereinfachend werden alle Leistungen als beitragsunabhängig betrachtet, so daß zur Ermittlung des äquivalenten Beitrags nur die Gesamtausgaben durch die Zahl der Versicherten geteilt werden müssen. Hieraus ergibt sich für 1989 ein äquivalenter monatlicher Beitrag von 191,36 DM. Es wird keine Risikodifferenzierung vorgenommen. ${ }^{45}$

\subsection{Empirischer Zusammenhang zwischen Netto-Äquivalenzeinkommen und Nettopo- sition}

Den folgenden Berechnungen liegen Daten aus dem Sozio-ökonomischen Panel zugrunde. Die verwendete Bruttostichprobe aus dem Jahr 1990 umfaßt 13.323 Personen in westdeutschen Haushalten bei 9.519 realisierten Interviews. Als GKV-Versicherte werden 11.114 Fälle einschließlich beitragsfrei mitversicherter Familienangehöriger betrachtet. Um zu repräsentativen Ergebnissen für die Gesamtbevölkerung zu gelangen, wurden die Hochrechnungsfaktoren des

42 Dieser Wert wurde aus der generierten Variable „GKV-Beitrag ohne Arbeitgeberanteil“ des SOEP durch Verdoppeln berechnet. Sowohl die generierte Variable als auch die Annahme, jedem GKV-Beitrag stünde ein gleich hoher Arbeitgeber-Beitrag oder ein Äquivalent (z.B. Beitrag des Rentenversicherungsträgers nach $\S 249$ a SGB V) hierfür gegenüber, geben die sozial- und finanzrechtliche Komplexität nur vereinfachend wieder (Ein solches Äquivalent fehlt z.B. bei freiwillig versicherten Rentnern ( $\$ 250$ Abs. SGB V)).

43 Vgl. hierzu Blankart, C.B., 1994, S.312f. und Euzéby, A., 1995.

44 Vgl. Statistisches Bundesamt, 1992b, S. 491.

45 Eine familienorientierte Versicherungsgestaltung derart, für die Kinder eine eigene Risikoklasse (mit dann niedrigerem äquivalenten Beitrag) zu bilden, würde zu einem höheren äquivalenten Beitrag für die Erwachsenen führen. 
Sozio-ökonomischen Panels benutzt. ${ }^{46}$ Die tatsächliche Beitragsbelastung wurde nicht Befragungsdaten entnommen (hier ergäbe sich das Problem, daß Befragte über ihre Beitragsbelastung oft irren), sondern wird mit einer aus der sozialversicherungsrechtlichen Beitragsbemessunggrundlage generierten Variablen gemessen.

Angesichts der Tatsache, daß die gesetzliche Krankenversicherung familienorientiert konstruiert ist, kann der Beitragzahlung des Haushaltsvorstands die beitragsfreie Mitversicherung meherer Familienangehöriger gegenüberstehen. Daher müssen im folgenden Haushalte als Betrachtungseinheit gewählt werden. Hierbei tritt das Problem auf, daß in einem Haushalt neben gesetzlich Krankenversicherten auch Privat- oder Nicht-Versicherte leben können. In diesen Fälle wird jeweils nur eine sogenannte "Versicherteneinheit" betrachtet, die aus dem GKVVersicherten im Haushalt besteht. Das Netto-Äquivalenzeinkommen der anderen Haushaltsmitglieder bleibt dann außer Betracht.

Wird nun das über die GKV-Versicherten in einem Haushalt summierte NettoÄquivalenzeinkommen zusammen mit der jeweiligen Nettoposition im sozialen Ausgleich in einem Sunflower-Plot dargestellt, ergibt sich folgendes Bild:

46 Für eine weiterführende Dokumentation des Sozio-ökonomische Panels vgl. Pannenberg, M./ Rendtel, U., 1996. 
Zusammenhang zwischen Haushalts-Nettoposition und summiertem Netto-Äquivalenzeinkommen, 1989 (DM/Monat)

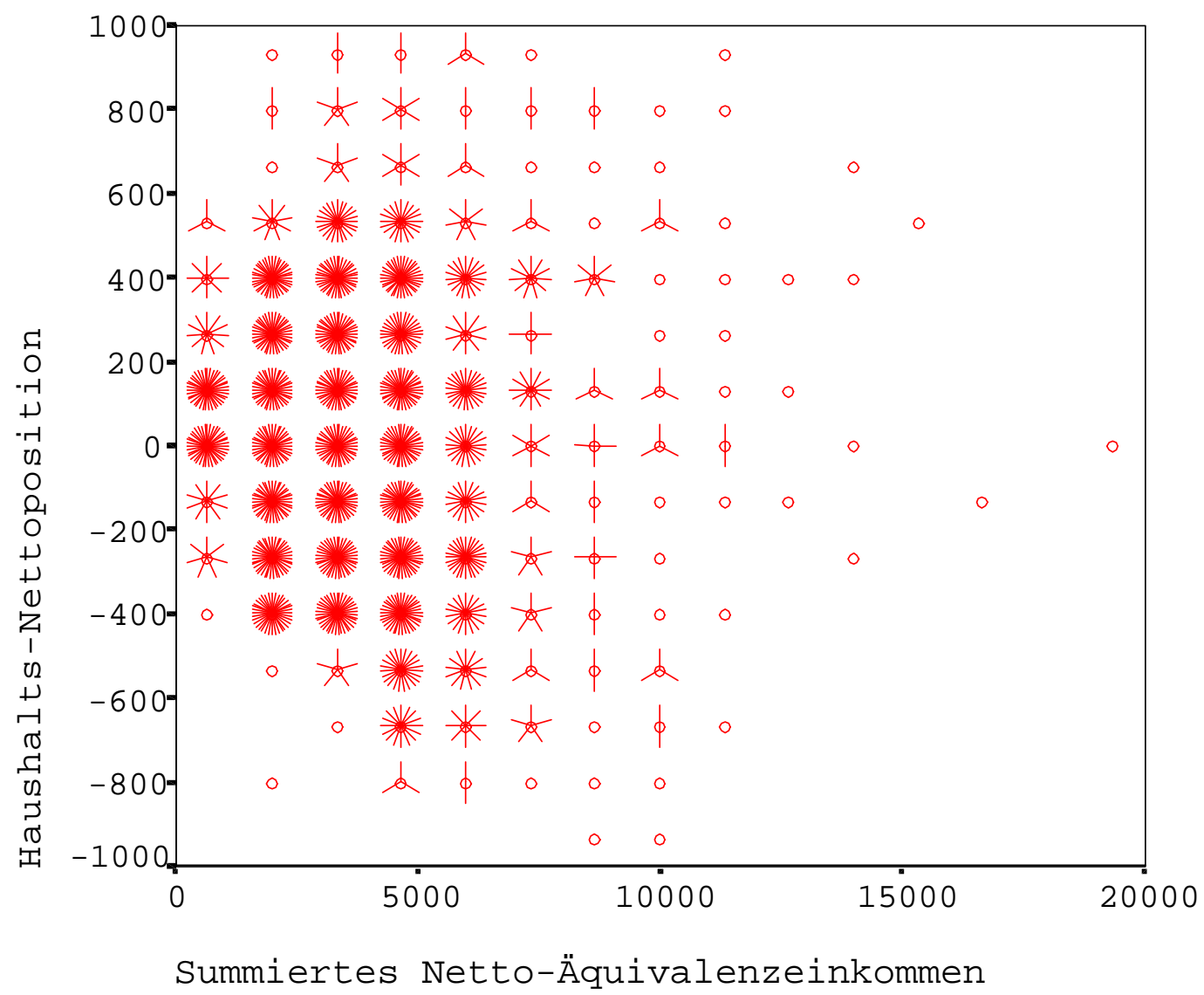

Jede Sunflower gibt die Zahl der Fälle in der sie umgebenden Region wieder. Ein "Blütenblatt" repräsentiert 10.000 Fälle. Schon der optische Eindruck weist auf eine nur geringe Korrelation hin. Der Pearson-Korrelationskoeffizient beträgt -0,047. Analog zeigt das Bestimmtheitsmaß $\mathrm{R}^{2}$ bei linearer Regression von nur 0,002, wie gering der Anteil der durch das NettoÄquivalenzeinkommen erklärten Varianz der Nettoposition an deren Gesamtstreuung ist. Dies deutet bereits darauf hin, daß der soziale Ausgleich sehr unabhängig von der sozialen Bedürftigkeit bzw. Leistungsfähigkeit der Haushalte wirkt.

\subsection{Horizontale und vertikale Effizienz}

Die Konzepte der horizontalen und vertikalen Effizienz sind geeignet, den empirischen Befund näher zu evaluieren. ${ }^{47}$ Horizontale Effizienz besteht, wenn alle Mitglieder der Zielgruppe ihrer Bedürftigkeit entsprechend begünstigt werden und es nicht zu ungerechtfertigten Auslassungen

47 Vgl. hierzu Atkinson, A.B., 1994, S.(3-7). 
kommt. Vertikale Effizienz ist gegeben, wenn sich eine sozialpolitische Maßnahme auf eine Zielgruppe (Bedürftige) konzentriert und es nicht zu Zuwendungen an Nicht-Bedürftige kommt.

Um das Konzept zum Zweck der Analyse des sozialen Ausgleichs zu operationalisieren, muß die Zielgruppe genauer abgegrenzt werden. Hierzu werden relative "Armutsgrenzen" verwendet, die ebenfalls auf das Netto-Äquivalenzeinkommen bezogen sind. ${ }^{48}$ Sie entsprechen 40, 50, und 60 v.H. des durchschnittlichen monatlichen Netto-Äquivalenzeinkommens und werden als relative Grenzen für "strenge Armut", "mittlere Armut" und "geringe Armut" bezeichnet. ${ }^{49}$

Schon die "geringe Armut" wird im folgenden als Eingriffsgrenze (EG) für einen sozialen Ausgleich gesetzt, um auch Grenzfälle sozialer Bedürftigkeit zu erfassen. Sie beginnt im betrachteten Kollektiv bei einem Netto-Äquivalenzeinkommen von 947,80 DM für 1989 für eine einzelne Person. Die Grenze vervielfacht sich für die Haushaltsbetrachtung mit der Anzahl der GKV-Versicherten im jeweiligen Haushalt. Die Berechnung des Äquivalenzeinkommens mit Hilfe der Regelsatzproportionen der Sozialhilfe sichert hierbei eine besondere Sensibilität für Familien mit Kindern.

Im ersten Schritt wird eine fiktive Situation "vor GKV" hergestellt. Dazu müssen zunächst die von den GKV-Versicherten $\mathrm{VS}_{1}$ bis $\mathrm{VS}_{\mathrm{s}}$ im Haushalt $\mathrm{j}$ tatsächlich geleisteten Beiträge ${ }^{50}\left(\mathrm{tB}^{\mathrm{V}}\right)$ zur gesetzlichen Krankenversicherung ihrem Netto-Äquivalenzeinkommen (NÄ $\ddot{\mathrm{V}}^{\mathrm{V}}$ zugerechnet werden. Die GKV-Beiträge (wie alle anderen Sozialabgaben) sind im HaushaltsNettoeinkommen, aus dem sich das Netto-Äquivalenzeinkommen berechnet, nicht enthalten. Damit ergibt sich das fiktive "Brutto"-Äquivalenzeinkommen $\left(B \ddot{A}^{\mathrm{HH}}\right)$ des Haushalts j (bzw. der GKV-Versicherten 1 bis s in diesem Haushalt) vor Zahlung der GKV-Beiträge als:

$$
\mathrm{B} \ddot{\mathrm{A}}_{\mathrm{j}}^{\mathrm{HH}}=\sum_{\mathrm{r}=1}^{\mathrm{s}} \mathrm{NA} \ddot{\mathrm{r}}_{\mathrm{r}}^{\mathrm{v}}+\sum_{\mathrm{r}=1}^{\mathrm{s}} \mathrm{tB}_{\mathrm{r}}^{\mathrm{V}}
$$

48 Vgl. z.B. Hochmuth, U./ Klee, G./ Volkert, J., 1995, S. 19 f. u. Hauser, R., 1995, S.4 f. Der Vorschlag einer relativen Grenze bei 50\% des Durchschnittseinkommens geht auf Fuchs, V., 1965 zurück.

49 Vgl. Statistisches Bundesamt, 1992a, S.482ff.

50 Der Arbeitgeberanteil wird wie in Gliederungspunkt 3.2 erläutert, als für die Beitragszahlung verwendeter Lohnbestandteil angesehen und ebenfalls eingerechnet. 
Im zweiten Schritt werden Beiträge zur Finanzierung der gesetzliche Krankenversicherung erhoben, jedoch ohne sozialen Ausgleich. Dazu werden von dem fiktiven "Brutto"Äquivalenzeinkommen $\left(\mathrm{B} \ddot{\mathrm{A}}^{\mathrm{HH}}\right.$ ) die zur Finanzierung des Versicherungschutzes der Haushaltsmitglieder erforderlichen äquivalenten Beiträge $\left(\ddot{a} \mathrm{~B}^{\mathrm{v}}\right)$ abgezogen. Daraus resultiert ein fiktives Netto-Äquivalenzeinkommen ( $\mathrm{fN} \ddot{\mathrm{A}}^{\mathrm{HH}}$ ) bei äquivalenten Beiträgen zur gesetzlichen Krankenversicherung:

$$
\mathrm{fN} \ddot{A}_{\mathrm{j}}^{\mathrm{HH}}=\mathrm{B} \ddot{\mathrm{A}}_{\mathrm{j}}^{\mathrm{HH}}-\sum_{\mathrm{r}=1}^{\mathrm{s}} \ddot{a ̈}_{\mathrm{r}}^{\mathrm{V}}
$$

Dies ist der Ausgangspunkt für eine Analyse der Bedürftigkeit in einem sozialen Ausgleich. Erst wenn die Aufbringung eines äquivalenten Beitrags für den in Anspruch genommen Versicherungsschutz den Haushalt überfordert, ist ein sozialpolitischer Eingriff gerechtfertigt.

In diesem Sinn werden die ausgleichsbedürftigen Haushalte und deren Ausgleichsbedarf bestimmt. Hierzu ist zunächst eine haushaltsbezogene Eingriffsgrenze $\left(\mathrm{EG}^{\mathrm{HH}}\right) \mathrm{zu}$ bestimmen. Sie ergibt sich aufgrund der üblichen Annahme von Haushalten als Einkommensgemeinschaften durch Summierung der Eingriffsgrenzen $\left(\mathrm{EG}^{\mathrm{V}}\right)$ für einzelne Personen über die Zahl der gesetzlich versicherten Haushaltsmitglieder:

$$
\mathrm{EG}_{\mathrm{j}}^{\mathrm{HH}}=\sum_{\mathrm{r}=1}^{\mathrm{s}} \mathrm{EG}_{\mathrm{r}}^{\mathrm{V}}
$$

Den Haushalten, die nach der Zahlung der äquivalenten Beiträge unter dieser Eingriffsgrenze der "geringen Armut" liegen, soll der Beitrag ermäßigt werden. Es kann maximal der gesamte Beitrag erlassen werden, jedoch darf die Ermäßigung höchstens die Einkommenslücke bis zur Eingriffsgrenze schließen. Der Ausgleichsbedarf (AB) beträgt somit:

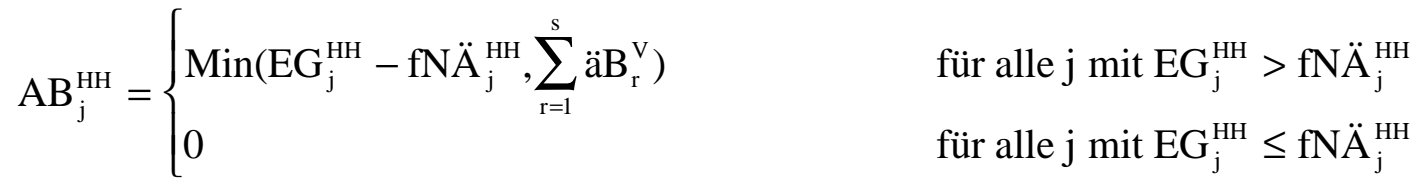

Nun wird der Beitrag des praktizierten sozialen Ausgleichs zur Deckung dieses Ausgleichsbedarfs ermittelt. Die Nettovorteile ausgleichsbedürftiger Haushalte $\left(\mathrm{NV}^{\mathrm{HH}}\right.$ mit $\mathrm{NV}^{\mathrm{HH}}=\mathrm{NP}^{\mathrm{HH}}$ für 
alle $\mathrm{NP}^{\mathrm{HH}}>0$ ) werden bis maximal zur Höhe ihres jeweiligen Ausgleichsbedarfs angerechnet und ergeben die Ausgleichsleistung (AL) an einen bedürftigen Haushalt:

$$
\mathrm{AL}_{\mathrm{j}}^{\mathrm{HH}}= \begin{cases}\operatorname{Min}\left(\mathrm{NV}_{\mathrm{j}}^{\mathrm{HH}}, \mathrm{AB}_{\mathrm{j}}^{\mathrm{HH}}\right) & \text { für alle } \mathrm{j} \text { mit } \mathrm{EG}_{\mathrm{j}}^{\mathrm{HH}}>\mathrm{fN} \ddot{A}_{j}^{\mathrm{HH}} \\ 0 & \text { für alle } \mathrm{j} \text { mit } \mathrm{EG}_{\mathrm{j}}^{\mathrm{HH}} \leq \mathrm{fN} \ddot{A}_{j}^{H H}\end{cases}
$$

Nettovorteile im praktizierten System, die den oben definierten Ausgleichsbedarf übersteigen, werden als Mehrleistungen an bedürftige Haushalte $\left(\mathrm{M}^{\mathrm{HH}}\right)$ bezeichnet:

$$
M_{j}^{\mathrm{HH}}= \begin{cases}\mathrm{NV}_{\mathrm{j}}^{\mathrm{HH}}-\mathrm{AB}_{\mathrm{j}}^{\mathrm{HH}} & \text { für alle } \mathrm{j} \text { mit } \mathrm{NV}_{\mathrm{j}}^{\mathrm{HH}}>\mathrm{AB}_{\mathrm{j}}^{\mathrm{HH}} \\ 0 & \text { für alle } \mathrm{j} \text { mit } N V_{j}^{\mathrm{HH}} \leq \mathrm{AB}_{j}^{\mathrm{HH}}\end{cases}
$$

Werden ausgleichsbedürftige Haushalte im praktizierten System nicht unterstützt, sondern haben statt dessen eine negative Nettoposition, kommt es zu armutsverschärfenden Nettozahlungen $\left(\mathrm{aNZ}^{\mathrm{HH}}\right)$.

$$
\mathrm{aNZ}_{\mathrm{j}}^{\mathrm{HH}}= \begin{cases}\mathrm{NP}_{\mathrm{j}}^{\mathrm{HH}} & \text { für alle } \mathrm{j} \text { mit } \mathrm{NP}_{\mathrm{j}}^{\mathrm{HH}}<0 \text { und } \mathrm{EG}_{\mathrm{j}}^{\mathrm{HH}}>\mathrm{fNÄ}_{\mathrm{j}}^{\mathrm{HH}} \\ 0 & \text { für alle j mit } \mathrm{NP}_{\mathrm{j}}^{\mathrm{HH}} \geq 0 \text { oder } \mathrm{EG}_{\mathrm{j}}^{\mathrm{HH}} \leq \mathrm{fNÄ}{ }_{j}^{\mathrm{HH}}\end{cases}
$$

Nicht-ausgleichsbedürftige Haushalte sind dadurch gekennzeichnet, einen äquivalenten Beitrag entrichten zu können, ohne unter die Grenze der "relativen Armut" zu fallen. Die Summe der ihnen gewährten Nettovorteile wird als fehladressierte Leistung $\left(\mathrm{fL}^{\mathrm{HH}}\right)$ bezeichnet.

$$
\mathrm{fL}_{\mathrm{j}}^{\mathrm{HH}}= \begin{cases}\mathrm{NP}_{\mathrm{j}}^{\mathrm{HH}} & \text { für alle } \mathrm{j} \text { mit } \mathrm{NP}_{\mathrm{j}}^{\mathrm{HH}}>0 \text { und } \mathrm{EG}_{\mathrm{j}}^{\mathrm{HH}}<\mathrm{fN}_{\mathrm{j}}^{\mathrm{HH}} \\ 0 & \text { für alle } \mathrm{j} \text { mit } \mathrm{NP}_{\mathrm{j}}^{\mathrm{HH}} \leq 0 \text { oder } \mathrm{EG}_{\mathrm{j}}^{\mathrm{HH}} \geq \mathrm{fN} \ddot{A}_{j}^{\mathrm{HH}}\end{cases}
$$

Die genannten haushaltsbezogenen Größen wurden im Datensatz ermittelt und mit Haushaltshochrechnungsfaktoren auf für Deutschland repräsentative Werte hochgerechnet. So ergeben sich Parameter für die Messung der sozialpolitischen Effizienz: 
Tabelle 1: Ergebnisse der Quantifizierung von Parametern zur Effizienzmessung im Jahr 1989

\begin{tabular}{|l|r|}
\hline Parameter zur Effizienzmessung & Mrd. DM \\
\hline 1. Ausgleichsbedarf & 21,656 \\
\hline 2. Ausgleichsleistung & 14,582 \\
\hline 3. Mehrleistungen & 1,472 \\
\hline 4. Fehladressierte Leistungen & 19,339 \\
\hline & \\
\hline \multicolumn{2}{|c|}{ Armutsverschärfende Nettozahlungen } \\
\hline \multicolumn{2}{|c|}{ 2/1 } \\
\hline Horizontale Effizienz & 3,941 \\
\hline Vertikale Effizienz & 0,67 \\
\hline
\end{tabular}

Eigene Berechnungen mit Daten des SOEP

Die Zahlen belegen die geringe sozialpolitische Effizienz des praktizierten sozialen Ausgleichs. Hervorzuheben ist besonders die äußerst geringe vertikale Effizienz, d.h. der hohe Anteil von Leistungen an nicht-bedürftige Haushalte. Auch werden keineswegs alle bedürftigen Haushalte durch den sozialen Ausgleich erreicht (geringe horizontale Effizienz). Außerdem werden einige in der Gruppe der Bedürftigen durch den sozialen Ausgleich sogar belastet statt entlastet. Andere wiederum werden überhaupt nur durch den sozialen Ausgleich unter die Armutgrenze gedrückt. Diese armutsverschärfenden Zahlungen betragen insgesamt knapp 4 Mrd. DM. AuBerdem bleibt ein Ausgleichsbedarf von über 7 Mrd. DM ungedeckt. Die Summe dieser Beträge bleibt jedoch noch weit unter der Höhe der fehladressierten Leistungen, die auch als "Streuverluste" des sozialen Ausgleichs bezeichnet werden könnten, von über 19 Mrd. DM.

\section{Fazit und Grundzüge eines Reformvorschlags}

Der soziale Ausgleich in der gesetzlichen Krankenversicherung verstößt gegen den finanzrechtlichen Grundsatz der Belastungsgleichheit, zeichnet sich durch eine geringe sozialpolitische Effektivität aus und erhöht im Vergleich zu steuerfinanzierten Lösungen die Personalzusatzkosten. 
Um das Prinzip der Belastungsgleichheit zum Tragen zu bringen, sollte in der gesetzlichen Krankenversicherung zum Äquivalenzprinzip zurückgefunden werden. ${ }^{51}$ Grundsätzlich wäre zunächst jeder Versicherte mit einem äquivalenten Beitrag zu belasten. ${ }^{52}$ Dort, wo der äquivalente Beitrag aus eigener Kraft nicht aufgebracht werden kann, könnte ein gezielter Transfer einsetzen. Im Jahr 1989 hätten dazu, legt man den in diesem Beitrag unter besonderer Berücksichtigung von Familien mit Kindern ermittelten Bedarf zugrunde, ca. 14 Mrd. DM aufgewendet werden müssen.

Ein solcher Transfer aus dem Steuerhaushalt würde Beitragszahlungen ersetzen und so die Personalzusatzkosten im Vergleich zur jetzigen Regelung insgesamt reduzieren. Der Transfer könnte auf Antrag gewährt und über die gesetzliche Krankenversicherung administriert werden. Dazu stünden verschiedene Wege offen:

- Eine Erstattung durch den Bund, wie dies bei den Leistungen nach $§ 200$ Reichsversicherungsordnung (Mutterschaftsgeld) bereits geschieht ( $\$ 200 \mathrm{a}$ Reichsversicherungsordnung).

- Ein Bundeszuschuß, wie er für die gesetzliche Rentenversicherung und die Bundesanstalt für Arbeit existiert (Bundeszuschüsse gibt es für die gesetzliche Krankenversicherung derzeit zwar nicht, sie sind jedoch verfassungsrechtlich in Art. 120 Abs. 1 Satz 4 vorgesehen).

- Transfers könnten aber auch ganz Geldleistungsgesetzen ("Medizingeld") vorbehalten werden. Diese Lösung käme am ehesten einem Systembruch nahe, da zunächst die in Rede stehenden Sozialleistungen im Bereich der GKV gestrichen und dann neue gesetzliche Sozialleistungsansprüche geschaffen würden.

Die Vergünstigungen könnten schließlich über eine sogenannte "Familienkasse", wie sie für die gesetzliche Rentenversicherung diskutiert wurde, finanziert werden. Dabei wird meist daran gedacht, diese aus der Umsatzsteuer zu speisen, die dann mit einer teilweisen Zweckbindung versehen werden würde.

51 Eine ähnliche Position vertritt auch der Sachverständigenrat zur Begutachtung der gesamtwirtschaftlichen Entwicklung, 1996, S.246 ff.

52 Damit ist nicht zwingend verbunden, die Arbeitgeberbeteiligung aufzugeben. Der Arbeitgeberbeitrag könnte wie oben gezeigt als zur Beitragszahlung verwendeter Lohnbestandteil auf den äquivalenten Beitrag angerechnet werden. 
Die drei erstgenannten Lösungen haben gemein, daß sie mit einer stärkeren Belastung des Haushaltes des Bundes, aufgrund des Kongruenzprinzips (Art 106 Abs. 4 GG) de jure auch der Haushalte der Länder, einhergehen. Diese Tatsache dürfte aufgrund der gegenwärtigen Haushaltslage in Bund und Ländern die politische Attraktivität dieses Reformansatzes stark beeinträchtigen. Auf der anderen Seite werden jedoch Ineffizienzen des oben beschriebenen AusmaBes nicht mehr lange finanzierbar sein. 


\section{Literatur}

Andel, Norbert, 1975: Verteilungswirkungen der Sozialversicherung am Beispiel der gesetzlichen Krankenversicherung in der Bundesrepublik Deutschland, in: Andel, Norbert/ Schneider Helmut (hrsg. von Wilhelmine Dreißig): Öffentliche Finanzwirtschaft und Verteilung III. Berlin: Duncker \& Humblot. Schriften des Vereins für Socialpolitik. Neue Folge Band 75/III. S.39-82.

Atkinson, Anthony B., 1994: On Targeting Social Security: Theory and Western Experience with Family Benefits, Dominique van de Walle, Kimberly Nead (eds.): Public Spending and the Poor. Theory and Evidence. Public Economics Division. Policy Research Department. The World Bank. January, pp. ( 3-1) - (3-59).

Atkinson, Anthony B., 1989: Poverty and Social Security. New York: Harvester Wheatsheaf.

Blankart, C. B., 1994: Öffentliche Finanzen in der Demokratie. München: Vahlen.

Buhmann, Brigitte/ Rainwater, Lee/ Schmaus, Guenther/ Smeeding, Timothy M., 1988: Equivalence Scales, Well-Being, Inequality, and Poverty: Sensitivity Estimates Across Ten Countries Using the Luxembourg Income Study (LIS) Database, in: Review of Income and Wealth, Vol. 34, pp.115-142.

Bundesministerium der Finanzen (Hrsg.), 1996: Finanzbericht 1997. Die volkswirtschaftlichen Grundlagen und wichtigsten finanzwirtschaftlichen Probleme des Bundeshaushaltsplans für das Haushaltsjahr 1997. Bonn: Bundesanzeiger Verlagsgesellschaft.

Bundestags-Drucksache 13/3339, 1995: Armut in der Bundsrepublik Deutschland. Antwort der Bundesregierung auf die große Anfrage der Abgeordneten Gilges, Gerd Andres, Ernst Bahr, weiterer Abgeordneter der Fraktion der SPD - Drucksache 13/1527 - zugeleitet am 13. Dezember 1995 über das Bundesministerium für Gesundheit

Becker, Irene, 1985: Einkommensumverteilung im Rahmen der gesetzlichen Krankenversicherung Eine empirische Untersuchung, in: Schmähl, Winfried (Hrsg.), Versicherungsprinzip und soziale Sicherung. Tübingen: Mohr.

Burkhauser, Richard V./ Duncan, Greg J./ Hauser, Richard, 1990: Sharing Prosperity Across the Age Distribution: A Comparison of the United States and Germany in the 1980s, in: The Gerontologist, Vol.34, No.2, pp. 150-160.

BVerfG 10.12.1980 E 55; 23.1.1990 E 81

Euzéby, Alain, 1995: Reduce or Rationalize Social Security Contributions to Increase Employment? In: International Labor Review 134, No. 2, pp. 227-241.

Fuchs, Victor, 1965: Toward a theory of poverty, in: Task Force on Economic Growth and Opportunity: The Concept of Poverty. Chamber of Commerce of the United States. Washington D.C.

Gesellschaft für Versicherungswissenschaften und -gestaltung e.V., 1992: Elemente eines Systems der Sozialen Sicherung unter den Bedingungen einer Sozialen Marktwirtschaft - Das Beispiel der Bundesrepublik Deutschland.

Hauser, Richard, 1995: Das empirische Bild der Armut in der Bundesrepublik Deutschland ein Überblick, in: Aus Politik und Zeitgeschichte. Beilage zur Wochenzeitung das Parlament B 31-32, 1995), S. 3-13.

Hauser, Richard/ Neumann, Udo, 1992: Armut in der Bundesrepublik Deutschland. Die sozialwissenschaftliche Thematisierung nach dem zweiten Weltkrieg, S. Leibfried/ W. Voges 
(Hrsg.): Armut im modernen Wohlfahrtsstaat. Kölner Zeitschrift für Soziologie und Sozialpsychologie, Sonderheft 32, S. 237-271.

Henke, Klaus-Dirk/ Behrens, Cornelia, 1989: Umverteilungswirkungen der gesetzlichen Krankenversicherung: eine empirische Analyse der differentiellen Einnahmewirkungen. Bayreuth: Verlag P.C.O. (Schriften zur Gesundheitsökonomie, Band 5).

Hochmuth, Uwe/ Klee, Günther/ Volkert, Jürgen, 1995: Armut in der Sozialen Marktwirtschaft. Möglichkeiten und Probleme ihrer Überwindung aus ordnungspolitischer Sicht. Tübingen und Basel: Francke Verlag (Tübinger volkswirtschaftliche Schriften; Bd 12).

Kirchhof, Ferdinand, 1994: Finanzierung der Krankenversicherung, in: Schulin, Bertram (Hrsg.): Handbuch des Sozialversicherungsrechts. Band 1 Krankenversicherungsrecht. München: Beck, S.1319-1331.

Kleinhenz, Gerhard, D., 1992: Die Zukunft des Sozialstaats. Spielraum für sozialen Fortschritt unter veränderten Rahmenbedingungen, in: Hamburger Jahrbuch für Wirtschafts- und Gesellschaftspolitik, 37. Jahr, Tübingen, S.43-71.

Lampert, Heinz, 1995: Voraussetzungen einer Sozialstaatsreform - kritische Anmerkungen zur aktuellen Diskussion über den Umbau des Sozialstaates, in: Jahrbücher für Nationalökonomie und Statistik, Bd. 214/5, S.513-531.

Männer, Leonhard,1997: Langfristige Versicherungsverhältnisse in der Gesetzlichen Krankenversicherung, in: Leonhard Männer (Hrsg.), Langfristige Versicherungsverhältnisse: Ökonomie, Technik, Institutionen. Karlsruhe: Verlag für Versicherungswirtschaft, S. 209-248.

Ott, G., 1981: Einkommensumverteilungen in der gesetzlichen Krankenversicherung. Frankfurt, Bern.

Pannenberg, Markus/ Rendtel, Ulrich, 1996: Dokumentation des Sozio-ökonomischen Panels (SOEP): Erhebungsdesign, Fallzahlen und erhebungsbedingte Ausfälle sowie die Schätzung von Auswahlwahrscheinlichkeiten bis Welle 12 (1984 bis 1995) [Stichprobe A,B und C]. Deutsches Institut für Wirtschaftsforschung. Diskussionspapier Nr.173a. Berlin, Juli 1996.

Sachverständigenrat zur Begutachtung der gesamtwirtschaftlichen Entwicklung, 1996: Reformen voranbringen. Jahresgutachten 1996/97. Bundestagsdrucksache 13/6200 vom 18.November.

Sachverständigenrat für die Konzertierte Aktion im Gesundheitswesen, 1996: Gesundheitswesen in Deutschland, Kostenfaktor und Zukunftsbranche. Band I. Bonn.

Schlenker, Rolf-Ulrich, 1994: Historische, sozialpolitische und ökonomische Grundlagen, in: Schulin, Bertram (Hrsg.): Handbuch des Sozialversicherungsrechts. Band 1 Krankenversicherungsrecht. München: Beck, S.1-47.

Schulin, Bertram, 1994: Rechtliche Grundprinzipien der gesetzlichen Krankenversicherung und ihre Probleme, in: Schulin, Bertram (Hrsg.): Handbuch des Sozialversicherungsrechts. Band 1 Krankenversicherungsrecht. München: Beck, S.177-248.

Statistisches Bundesamt (Hrsg.), 1992a: Datenreport 1992. Zahlen und Fakten über die Bundesrepublik Deutschland. Bundeszentrale für politische Bildung. (Schriftenreihe, Band 309. Arbeitshilfen für die politische Bildung).

Statistisches Bundesamt (Hrsg.), 1992b: Statistisches Jahrbuch 1992 für die Bundesrepublik Deutschland. Stuttgart: Metzler-Poeschel.

Statistisches Bundesamt (Hrsg.), 1996: Fachserie 13 Sozialleistungen. Reihe 1 Versicherte in 
der Kranken- und Rentenversicherung 1995. Stuttgart: Metzler-Poeschel.

Statistisches Bundesamt (Hrsg.), 1997: Statistisches Jahrbuch 1997 für die Bundesrepublik Deutschland. Stuttgart: Metzler-Poeschel.

Wagner, Gert, 1997: Steine auf dem Weg zu einer effizienten Krankenversicherung, in: Tagungsband zur Jahresversammlung der Arbeitsgemeinschaft deutscher wirtschaftswissenschaftlicher Forschungsinstitute, Bonn, April 1997. Beihefte zur Konjunkturpolitik, Heft 46, S. 89115.

Wasem, Jürgen, 1994: Sozialpolitische Grundlagen der gesetzlichen Krankenversicherung, in: Schulin, Bertram (Hrsg.): Handbuch des Sozialversicherungsrechts. Band 1 Krankenversicherungsrecht. München: Beck, S.80-112.

Zimmermann, Horst, Henke, Klaus-Dirk, 1994: Finanzwissenschaft. Eine Einführung in die Lehre von der öffentlichen Finanzwirtschaft, 7. Aufl., München: Vahlen. 